\title{
Comparative Study for Clinical Outcomes of Triple-Positive and Triple- Negative Breast Cancer: Long-term Results in 161 Patients Followed in a Single Center
}

\author{
Seungyeol Baeg, M.D. ${ }^{1}$, Inseok Park, M.D. ${ }^{1}$, Jungbin Kim, M.D. ${ }^{1}$, Chansub Park, M.D. ${ }^{5}$, Hyunjin Cho, M.D. ${ }^{1}$, \\ Keunho Yang, M.D., Ph.D. ${ }^{1}$, Jiyoung Kim, M.D., Ph.D. ${ }^{3}$, Youngjoo Shin, M.D., Ph.D. ${ }^{4}$, Kyeongmee Park, M.D., Ph.D. ${ }^{2}$ \\ Geumhee Gwak, M.D., Ph.D. ${ }^{1}$ \\ ${ }^{1}$ Department of Surgery, ${ }^{2}$ Department of Pathology, ${ }^{3}$ Department of Radiology, ${ }^{4}$ Department of Radiation Omncology, Sanggye Paik Hospital, Inje \\ University, College of Medicine, Seoul; ${ }^{5}$ Department of Breast and Thyroid Surgery, Kyungpook National University Chilgok Hospital, Daegu, Korea
}

Purpose: Triple negative breast cancer (TNBC) is one of the most aggressive subtypes of breast cancer. However, we have often experienced that triple positive breast cancer (TPBC) shows more aggressive clinical features than TNBC. In this retrospective study, we aimed to examine the differences in clinical courses between TNBC and TPBC. Methods: Using medical records and clinical data, we selected patients with breast cancer who met the criteria for the two groups, TNBC and TPBC, based on the expression or absence of the estrogen receptor (ER), progesterone receptor (PR), and human epidermal growth factor receptor-2 (HER2). We then compared these groups with respect to clinical and pathological variables, such as patient age at diagnosis, TNM stage, number of tumors, involvement of resection margin, operation methods, histologic grade (HG), nuclear grade (NG), and lymphatic invasion (LI). We also compared the disease-free (DFS) and overall survival (OS) outcomes between the groups. Results: Seventy patients with TNBC and 91 with TPBC were identified among a total of 628 patients. In univariate analysis, TPBC was significantly more frequently associated with lower HG ( $p=0.001)$, lower NG ( $p=0.003)$, LI $(p=0.001)$, and a Ki-67 index $\leq 20 \%(p<0.001)$. In multivariate analysis, a lower Ki-67 index $(p=0.031)$ and $\mathrm{LI}(p=0.022)$ were identified as significant and independent factors contributing to DFS. In a survival analysis over time, the TPBC showed a worse OS than TNBC 5 years post-treatment for breast cancer. Consequently, the TPBC group had definite worse 10-year DFS $(p=0.012)$ and showed relatively lower OS rate $(p=0.058)$, than the TNBC group. Conclusion: Our results demonstrate considerable differences in long-term post-treatment survival of patients with TPBC and TNBC. Further studies to determine the proper management of both types of breast cancer and an accurate prognostic evaluation method are warranted.

Key Words: Breast neoplasms, Prognosis, Survival

\section{INTRODUCTION}

Breast cancer is a highly heterogeneous disease caused by disruptions in various regulators of intracellular signaling pathways. Three biological markers, namely the estrogen receptor (ER), progesterone receptor (PR), and human epidermal growth factor receptor-2 (HER2), are considered the principal factors in the dysregulated signaling pathways associated with breast cancer [1,2]. In addition, key information about the biological characteristics of this malignancy can be obtained from immunohistochemical (IHC) studies of these

Correspondence: Geumhee Gwak, M.D., Ph.D.

Department of Surgery, Sanggye Paik Hospital, Inje University College of Medicine, 1342 Dongil-ro, Nowon-gu, Seoul 01757, Korea

Tel: +82-2-950-1017, Fax: +82-2-933-9389, E-mail: ggh2008@paik.ac.kr

Received: Aug 30, 2019 Revised: Nov 4, 2019 Accepted: Mar 1, 2020 three markers. Approximately $60-80 \%$ of breast cancers express ER and/or PR [3], while approximately 20-30\% overexpress HER2/neu. More recently, however, gene expression profiling has been used to develop molecular classifications of five major breast cancer subtypes: luminal A, luminal B, HER2, basal-like, and normal breast-like [4]. Although researchers initially thought that HER2 positivity was associated with ER and/or PR negativity, further studies conducted in a clinical setting revealed that approximately half of HER2-positive breast cancers exhibited some level of ER and/or PR positivity [5]. More specifically, nearly half of all HER2 positive breast cancers simultaneously express ER and PR. These lesions comprise the subset of triple-positive breast cancers (TPBCs), which are largely consistent with the category of HER2-positive luminal B breast cancer.

Generally, triple-negative breast cancer (TNBC) is associated with 
the most aggressive behavior and worse long-term clinical outcomes, compared to other breast cancer subtypes [6,7]. However, TPBC has been found to exhibit a more aggressive clinical behavior than HER2-negative luminal A breast cancer, despite the availability of chemotherapy, anti-HER2 targeted therapy, and anti-estrogen therapy for patients with the former subtype [8]. Notably, several retrospective long-term follow-up studies of patients with early-stage ER- and/ or PR-positive, HER2-negative breast cancer revealed that luminal-subtype early breast cancer confers a residual risk of distant recurrence after 5 years of endocrine therapy. Moreover, the luminal B subtype is well known to have worse 5- and 10-year distant recurrence-free survival rates, compared to the luminal A subtype. Furthermore, we observed that the survival curves of patients with luminal B subtype and basal-like disease crossed each other after approximately 10 years of follow-up $[9,10]$, and two large studies showed that among HER2-positive breast cancers, hormone receptor (HR)-negative disease was associated with a higher incidence of cancer relapse within 5 years than HR-positive disease $[11,12]$.

HER2/neu overexpression negatively affects the response of a HR-positive breast cancer to tamoxifen therapy, as demonstrated by Houston et al. who reported that among patients with advanced ER-positive breast cancer, those with HER2-positive breast cancer exhibited a significantly shorter time to progression after receiving tamoxifen [13]. Additionally, HER2/neu overexpression is closely linked with a higher rate of recurrence, despite proper surgical management and adjuvant chemotherapy [14]. Therefore, we explored whether TPBC patients have an increased frequency of late recurrence due to HR-positive disease after 5 years of follow-up and whether TNBC is associated with a higher frequency of early recurrence due to HR-negative disease before 5 years of follow-up. Further, we also aimed to evaluate the long-term (10-year) prognosis of patients with TPBC and TNBC.

\section{METHODS}

For this study, we retrospectively collected the medical records and clinical data of 628 patients with breast cancer who were diagnosed at a single medical institute between January 1,2004 and September 20, 2012. After excluding patients with stage 0 disease, which has only minimal associations with survival and recurrence, we then used ER,
PR, and HER2 expression data to identify patients with TNBC and TPBC. TPBC was defined as ER and PR positivity detected via IHC assays and a HER2 IHC score of 3+ or HER2 gene amplification detected by fluorescence in situ hybridization (FISH) or silver in situ hybridization (SISH) of breast cancer tissue. TNBC was defined by the lack of ER and PR expression in IHC assays and a HER2 IHC score of 0 or $1+$ or no HER 2 amplification via SISH or FISH.

We compared clinical and pathological variables, such as patient age at diagnosis, TNM stage, number of tumors, margin involvement, operative method, histological grade (HG), nuclear grade (NG), lymphatic invasion (LI), vascular invasion (VI), neural invasion (NI), Ki67 index, and Bcl-2 expression, between the TNBC and TPBC groups. We also compared follow-up data between the groups, such as recurrence events, death, and metastasis to the breast, lymph node, bone, lung, liver, brain, and other visceral organs. Furthermore, we compared the disease-free survival (DFS) and overall survival (OS) between patients with TNBC and TPBC. This study was approved by the Institutional Review Board (IRB No. 2017-01-012-002) and met the ethical standards of the Declaration of Helsinki.

\section{IHC staining for ER, PR, Bcl-2, and $\mathrm{Ki}-67$}

Mouse monoclonal antibodies specific for ER (NCL-1-ER-6F11) and PR (NCL-L-PGR-312; both Leica Microsystems Inc. clone code numbers, Newcastle Upon Tyne, UK), were diluted at 1:80 in a solution of $20 \%$ normal goat serum (in Tris-buffered saline; TBS) and used as primary antibodies for ER and PR IHC staining, respectively. Subsequently, a peroxidase-conjugated goat anti-mouse immunoglobulin was used as the secondary antibody. After treatment with the chromogen 3, 3'-diaminobenzidine tetrahydrochloride (DAB), ER, and PR staining intensities were scored as $0,1+, 2+$, and $3+$, together with a description of the percentage related to the proportion of stained nuclei in 10 high-power fields. The intensity scores and percentages were then converted into Allred scores, where a score of 0 was defined as negative, $1-6$ as weakly positive, and 7-8 as strongly positive. Here, we defined an Allred score $>6$ for both ER and PR as strongly positive for both ER and PR.

IHC for Bcl-2 and Ki-67 was performed using the avidin-biotin peroxidase complex method, with the Vectastain ABC Elite kit (Vector Laboratories, Burlingame, USA) and aminoethylcarbazole as the chromogen. Bcl-2 overexpression was defined as a Bcl-2 staining in- 
tensity $>33 \%$. A Ki-67 cut-off of $20 \%$ was used to identify the low proliferative group, which was considered optimal when stratifying highrisk patients with luminal breast cancer.

\section{IHC staining for HER2/neu}

Primary antibody binding was detected using the Dako QuickStaining, Labelled Streptavidin-Biotin System (Dako, Carpinteria, USA), followed by the DAB chromogen. Each slide was scored in a blinded fashion by two pathologists according to the manufacturer's recommended criteria. Intensity scores of 0 or $1+$ were considered negative for HER2/neu, while a score of $3+$ was considered positive. A score of $2+$ was considered equivocal, and the tissue was subjected to silver-enhanced in situ hybridization (SISH)[15].

\section{SISH for HER2}

HER2 SISH was performed using a Ventana Benchmark automated instrument (Ventana Medical Systems Inc., Tucson, USA) according to the manufacturer's protocols for the INFORM HER2 DNA and chromosome 17 probes. The evaluation of HER2 gene amplification was performed in a blinded manner according to the guidelines of the American society of clinical oncology/college of American pathologists [16].

\section{Statistical methods}

The chi-square test was used to analyze clinicopathological factors affecting the prognosis of patients in the TPBC and TNBC group. Differences in DFS and OS between the two groups were analyzed using the Kaplan-Meier method, and a Cox multivariate regression model was used for the multivariate analysis. SPSS Statistics version 21 (IBM Corp., Armonk, USA) and MedCalc Statistical Software version 17.6 (MedCalc Software, Ostend, Belgium) were used for statistical analyses. A $p$-value $<0.05$ was considered to indicate statistical significance.

\section{RESULTS}

\section{Demographic and clinical characteristics of patients} with TNBC and TPBC

Demographic and clinical characteristics of 70 patients with TPBC and 91 patients with TNBC are shown in Table 1. A univariate analy-
Table 1. Demographic and clinical characteristics of TPBC and TNBC patients

\begin{tabular}{|c|c|c|c|c|}
\hline \multicolumn{2}{|l|}{ Characteristic } & \multirow{2}{*}{$\begin{array}{c}\text { TPBC } \\
(n=70) \\
\text { No. }(\%) \\
42(60.0)\end{array}$} & \multirow{2}{*}{$\begin{array}{c}\text { TNBC } \\
(n=91) \\
\text { No. }(\%) \\
48(52.7)\end{array}$} & \multirow{2}{*}{$\begin{array}{c}p \text {-value } \\
0.360\end{array}$} \\
\hline Age (yr) & $\leq 50$ & & & \\
\hline & $>50$ & $28(40.0)$ & $43(47.3)$ & \\
\hline \multirow[t]{4}{*}{ TNM stage } & I & $19(27.1)$ & $33(36.3)$ & 0.634 \\
\hline & $\|$ & $34(48.6)$ & 41 (45.1) & \\
\hline & III & $15(21.4)$ & $15(16.5)$ & \\
\hline & IV & $2(2.9)$ & $2(2.2)$ & \\
\hline \multirow[t]{3}{*}{ Nodal status } & 0 & $30(43.7)$ & $63(68.9)$ & 0.006 \\
\hline & $1-3$ & 21(29.6) & $15(16.7)$ & \\
\hline & $\geq 4$ & $19(26.8)$ & $13(14.4)$ & \\
\hline \multirow[t]{2}{*}{ Stage group } & $\mathrm{I}, \mathrm{II}$ & $53(75.7)$ & $74(81.3)$ & 0.389 \\
\hline & III, IV & $17(24.3)$ & $17(18.7)$ & \\
\hline \multirow[t]{2}{*}{ Breast operations } & $\mathrm{BCS}$ & $47(67.1)$ & $63(69.2)$ & 0.778 \\
\hline & Mastectomy & $23(32.9)$ & $28(30.8)$ & \\
\hline \multirow[t]{2}{*}{ Axilla operations } & SLNB & $20(28.6)$ & $36(39.6)$ & 0.186 \\
\hline & ALND & $50(71.4)$ & $55(60.4)$ & \\
\hline \multirow[t]{2}{*}{ Operations } & BCS & $54(66.7)$ & $64(70.3)$ & 0.606 \\
\hline & Mastectomy & $27(33.3)$ & $27(29.7)$ & \\
\hline \multirow[t]{2}{*}{ Chemotherapy } & No & $3(4.3)$ & $8(8.8)$ & 0.263 \\
\hline & Yes & $67(95.7)$ & $83(91.2)$ & \\
\hline \multirow[t]{2}{*}{ Radiation therapy } & No & $32(45.7)$ & $43(47.3)$ & 0.847 \\
\hline & Yes & $38(54.3)$ & $48(52.7)$ & \\
\hline \multirow[t]{2}{*}{ Endocrine therapy } & No & $7(10.0)$ & 85 (93.4) & $<0.001$ \\
\hline & Yes & $63(90.0)$ & $6(6.6)$ & \\
\hline \multirow[t]{2}{*}{ HER2 Targeted therapy } & No & $45(64.3)$ & 90 (98.9) & $<0.001$ \\
\hline & Yes & $25(35.7)$ & $1(1.1)$ & \\
\hline
\end{tabular}

$\mathrm{TPBC}=$ triple positive breast cancer; $\mathrm{TNBC}=$ triple negative breast cancer; $\mathrm{BCS}=$ breast conserving surgery; $\mathrm{SLNB}=$ sentinel lymph node biopsy; $\mathrm{ALND}=$ axillary lymph node dissection; HER2 = human epidermal growth factor 2 .

sis revealed similar proportions of patients with advanced-stage TPBC and TNBC (24.3\% vs. 18.7\%). Twenty-five (35.7\%) and 63 (90.0\%) patients with TPBC received HER2 targeted therapy and endocrine therapy, respectively. Pathological characteristics of patients with TPBC and TNBC are shown in Table 2. Here, a univariate analysis revealed significant associations of a lower HG $(p=0.001)$, lower NG ( $p=0.003)$, the presence of LI $(p=0.001)$, and NI $(p=0.002)$, a higher Bcl-2 intensity $(p<0.001)$, and lower Ki-67 index $(p<0.001)$ with TPBC. However, no significant intergroup differences were observed with respect to the age at diagnosis, TNM stage, number of tumors, margin involvement, operative method, and the presence of VI.

Organ-specific recurrences of TPBC and TNBC are shown in Table 3. Although the presence of bone metastasis $(p=0.001)$ was found to be significantly associated with TPBC, no significant associations were observed with liver, lung, and brain metastases. In a multivariate analysis, lower Ki-67 index ( $p=0.031)$ and the presence of LI ( $p=$ 
Table 2. Pathologic characteristics of TPBC and TNBC

\begin{tabular}{|c|c|c|c|c|}
\hline \multicolumn{2}{|l|}{ Characteristic } & $\begin{array}{c}\text { TPBC } \\
(n=70) \\
\text { No. }(\%)\end{array}$ & $\begin{array}{c}\text { TNBC } \\
(n=91) \\
\text { No. }(\%)\end{array}$ & $p$-value \\
\hline \multirow{3}{*}{ Number of tumor } & & & & \\
\hline & Multiplo & (15) & ) & \\
\hline & Multıple & $11(15 . /)$ & $9(9.9)$ & \\
\hline \multirow[t]{2}{*}{ Resection margin } & Clear & $66(94.3)$ & $86(94.5)$ & 0.952 \\
\hline & Involvement & $4(5.7)$ & $5(5.5)$ & \\
\hline \multirow[t]{4}{*}{$H G$} & I & $2(2.9)$ & 0 & 0.001 \\
\hline & $\|$ & $16(22.9)$ & $4(4.4)$ & \\
\hline & III & $46(65.7)$ & 77 (84.6) & \\
\hline & $\mathrm{N} / \mathrm{A}$ & $6(8.6)$ & $10(11.0)$ & \\
\hline \multirow[t]{4}{*}{ NG } & I & $42(60.0)$ & $71(78.0)$ & 0.003 \\
\hline & $\|$ & $20(28.6)$ & $6(6.6)$ & \\
\hline & III & $2(2.9)$ & $4(4.4)$ & \\
\hline & $\mathrm{N} / \mathrm{A}$ & $6(8.6)$ & $10(11.0)$ & \\
\hline \multirow[t]{2}{*}{ LI } & No & $23(32.9)$ & $53(58.2)$ & 0.001 \\
\hline & Yes & $47(67.1)$ & $38(41.8)$ & \\
\hline \multirow[t]{2}{*}{$\mathrm{VI}$} & No & $55(78.5)$ & $80(87.9)$ & 0.112 \\
\hline & Yes & $15(21.4)$ & $11(12.1)$ & \\
\hline \multirow[t]{2}{*}{$\mathrm{NI}$} & No & $63(90.0)$ & $91(100.0)$ & 0.002 \\
\hline & Yes & $7(10.0)$ & 0 & \\
\hline \multirow[t]{2}{*}{ Ki 67} & $\leq 20 \%$ & 41 (58.6) & $7(7.7)$ & $<0.001$ \\
\hline & $>20 \%$ & $29(41.4)$ & $84(92.3)$ & \\
\hline \multirow[t]{2}{*}{$\mathrm{BCl}-2$} & $\leq 30 \%$ & $21(30.0)$ & 74 (81.3) & $<0.001$ \\
\hline & $>30 \%$ & $49(70.0)$ & $17(18.7)$ & \\
\hline
\end{tabular}

$\mathrm{TPBC}=$ triple positive breast cancer; $\mathrm{TNBC}=$ triple negative breast cancer; $\mathrm{HG}=$ histologic grade; $\mathrm{NG}=$ nuclear grade; $\mathrm{N} / \mathrm{A}=$ Not available; $\mathrm{LI}=$ lymphatic invasion; $\mathrm{Vl}=$ vascular invasion; $\mathrm{Nl}=$ neural invasion.

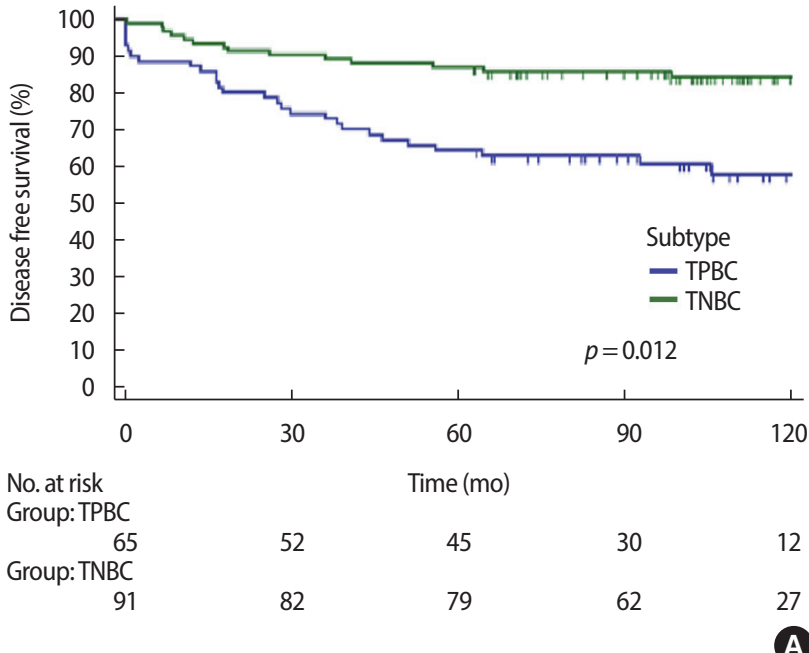

Table 3. Organ specific recurrences of TPBC and TNBC

\begin{tabular}{|c|c|c|c|c|}
\hline \multicolumn{2}{|l|}{ Variable } & \multirow{2}{*}{$\begin{array}{c}\text { TPBC } \\
(n=70) \\
\text { No. }(\%) \\
62(88.6)\end{array}$} & \multirow{2}{*}{$\begin{array}{c}\text { TNBC } \\
(n=91) \\
\text { No. }(\%) \\
87(95.6)\end{array}$} & \multirow{2}{*}{$\begin{array}{c}p \text {-value } \\
0.093\end{array}$} \\
\hline Breast & No & & & \\
\hline & Yes & $8(11.4)$ & $4(4.4)$ & \\
\hline \multirow[t]{2}{*}{ Bone } & No & 54 (77.1) & $86(94.5)$ & 0.001 \\
\hline & Yes & $16(22.9)$ & $5(5.5)$ & \\
\hline \multirow[t]{2}{*}{ Lung } & No & $63(90.0)$ & 85 (93.4) & 0.433 \\
\hline & Yes & $7(10.0)$ & $6(8.6)$ & \\
\hline \multirow[t]{2}{*}{ Liver } & No & $63(90.0)$ & 89 (97.8) & 0.033 \\
\hline & Yes & $7(10.0)$ & $2(2.2)$ & \\
\hline \multirow[t]{2}{*}{ Brain } & No & 65 (92.9) & 89 (97.8) & 0.128 \\
\hline & Yes & $5(7.1)$ & $2(2.2)$ & \\
\hline \multirow[t]{2}{*}{ LN } & No & 64 (91.4) & 83 (91.2) & 0.961 \\
\hline & Yes & $6(8.6)$ & $8(8.8)$ & \\
\hline
\end{tabular}

$\mathrm{TPBC}=$ triple positive breast cancer; $\mathrm{TNBC}=$ triple negative breast cancer.

Table 4. Multivariate analysis for prognostic factors related DFS and overall survival

\begin{tabular}{lcc}
\hline Variable & Odds ratio $(95 \% \mathrm{Cl})$ & $p$-value \\
\hline $\begin{array}{l}\text { Disease free survival } \\
\text { Ki-67 }\end{array}$ & $0.41(0.19-0.92)$ & 0.031 \\
LI & $2.58(1.15-5.79)$ & 0.022 \\
Overall survival & & \\
Bone metastasis & $75(18.98-296.43)$ & $<0.001$ \\
\hline
\end{tabular}

$\mathrm{Cl}=$ confidence interval, $\mathrm{DFS}=$ disease free survival, $\mathrm{LI}=$ lymphatic invasion.

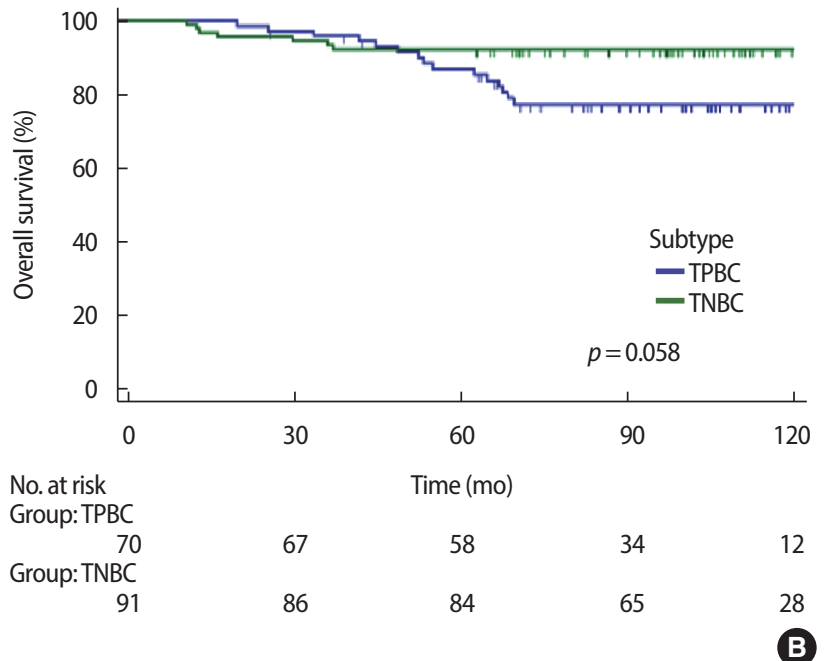

Figure 1. (A) 10-year disease free survival (DFS) and (B) overall survival (OS) for TNBC and TPBC.

0.022) were identified as significant and independent prognostic factors for DFS, with respective odds ratios ( $95 \%$ confidence intervals; CIs) of 0.41 (0.19-0.92) and 2.58 (1.15-5.79) (Table 4). Moreover, bone metastasis was identified as a significant and independent prognostic factor for OS $(p<0.001)$, with an odds ratio $(95 \%$ CI) of 75 (18.98296.43) (Table 4). 
Ten-year DFS and OS outcomes in patients with TNBC and TPBC

TNBC was associated with a significantly better DFS than TPBC, as the former was associated with a $15 \%$ lower recurrence rate at a 10 year follow-up $(p=0.012)$ (Figure 1A). Moreover, TNBC showed a better tendency on 10 -year OS ( $p=0.058)$. Analysis of the survival curves for TPBC and TNBC revealed a crossover point at approximately 5 years after breast cancer diagnosis (Figure 1B).

\section{DISCUSSION}

Precise evaluations of the ER and PR statuses, HER2 gene amplification, and the Ki-67 index are core processes used to predict the therapeutic responses and prognoses of individual patients with breast cancer [17]. However, patients with TPBC may benefit from anti-estrogen and anti-HER2 therapies, whereas those with TNBC have limited therapeutic options (e.g., cytotoxic chemotherapy). Results from many studies suggest that anti-HER2 therapy can prolong DFS and OS in patients with HER2-positive breast cancer [18]. However our clinical observations indicate a relatively high frequency of longterm disease recurrence and progression despite radical breast cancer treatment in this subset of patients.

Accordingly, we conducted this retrospective study to present a unique perspective on the long term prognosis of TPBC and TNBC patients, with a median follow up time of 86 months. Notably, we found that patients with TPBC were more likely to relapse than those with TNBC. Interestingly, the OS curve of the TPBC group decreased compared to that of the TNBC group after approximately 5 years of follow-up, suggesting that patients in the former group had a relatively lower rate of survival beyond 5 years. In other words, in real-world settings, TPBC may be more challenging than TNBC.

During the study period, a standard period of 5 years of hormonal therapy was recommended for patients with HR-positive breast cancer who had completed definitive surgery and/or adjuvant chemotherapy. However, to accommodate the relatively high frequency of late relapses of HR-positive breast cancers, and following the recommendations of multiple large-scale clinical trials, more recent guidelines have been developed to increase the duration of hormonal therapy to 10 years [19]. This may explain why the survival curves of TPBC continues to decline after five years. We speculate that our results may have been different if TPBC patients had received appropriate anti-HER2 therapy and extended hormonal therapy. Therefore, further studies involving patients receiving proper management for TNBC and TPBC, as recommended by recent guidelines, are needed to evaluate the prognosis of each group.

Our comparative analysis revealed that relative to TNBC, TPBC was more likely to be associated with a low HG, frequent LI and NI, higher Bcl-2 expression, and a lower Ki-67 index. These findings contrast directly with the results of previous studies comparing the prognoses of both groups. For example, both Onitilo et al. [20] and Negi et al. [21] compared the prognoses of TNBC and TPBC based on molecular subtyping, and concluded that TNBC had a relatively poorer prognosis, despite proper management. However, data from both of these studies suggested that patients with TNBC were diagnosed at a younger age and had a higher tumor stage, which may have had serious effects on prognosis. Many other studies have reported that, compared to patients with other breast cancer subtypes, patients with TNBC tend to be diagnosed at a younger age and to have a high $\mathrm{HG}$ and a more advanced disease stage $[22,23]$.

In our study, we found that the TNBC group tended to be associated with a higher HG, lower Bcl-2, higher Ki67 index, and more advanced TNM stage. The Ki-67 index is a well-known independent prognostic factor of both DFS and OS in patients with breast cancer [24]. Consequently, the Ki-67 index can be used in combination with other biological markers to determine the molecular subtype [25]. Although we expected to observe a poorer clinical course in the TNBC group than in the TPBC group, based on the prognostic impact of the Ki-67 index, we instead observed a better prognosis for patients with a high Ki-67 index (i.e., TNBC) in terms of long term survival outcomes with a median follow up time of 86 months. Although the exact impact of the Ki-67 index on each breast cancer subtype remains uncertain, we presume that this factor has a weaker prognostic impact in patients with TPBC than in those with TNBC. Moreover, a strong expression of $\mathrm{Bcl}-2$, an inhibitor of cell apoptosis frequently expressed in breast cancer, has been reported by multiple studies as being linked with HR positivity and improved survival $[26,27]$. As expected, we observed significantly higher Bcl-2 expression in TNBC than in TPBC, as the latter is ER- and PR-positive.

Previous studies have yielded consistent patterns of breast cancer, which are often associated with the HR status. Clinically, HR-positive 
breast cancer is associated with a higher incidence of bone, soft tissue, and gonadal metastases, whereas HR-negative breast cancer tends to generate life-threatening metastases of organs such as the brain and liver [14]. Our analysis clearly demonstrates that bone metastasis occurred more frequently in patients TPBC. However the rates of liver, brain and other distant metastases and also of loco-regional recurrence were similar in both groups.

Our study had some potential limitations. First, our study population comprised only a small number of patients who were sorted from a small patient pool at a single institute. Accordingly, our analysis of potentially meaningful prognostic factors of breast cancer may have been weak in terms of statistical power. Second, only 26 of 81 patients in the TPBC group received targeted therapy. In other words, two thirds of patients with TPBC received a suboptimal treatment, which might have affected the analysis of long-term prognosis in this group.

In conclusion, our study findings demonstrate that at approximately 5 years after diagnosis, the survival rate of patients with TPBC tended to decrease below that of patients with TNBC. The TPBC group involved the more spinous subtype of breast cancer, which requires a more delicate treatment plan including optimal anti-hormonal therapy and anti-HER-2 therapy. Given that our study included only a small group of patients belonging to a single center, further large-scale clinical trials are needed to validate our results.

\section{CONFLICT OF INTEREST}

The authors declare that they have no competing interests.

\section{REFERENCES}

1. Kamdje AHN, Etet PFS, Vecchio L, Muller JM, Krampera M, Lukong KE. Signaling pathways in breast cancer: therapeutic targeting of the microenvironment. Cell Signal 2014;26:2843-56.

2. The cancer genome atlas network. Comprehensive molecular portraits of human breast tumours. Nature 2012;490:61-70.

3. Clark GM, Osborne CK, McGuire WL. Correlations between estrogen receptor, progesterone receptor, and patient characteristics in human breast cancer. JClin Oncol 1984;2:1102-9.

4. Perou CM, Sorlie T, Eisen MB, van de Rijn M, Jeffrey SS, Rees CA, et al. Molecular portraits of human breast tumours. Nature 2000;
406:747-52.

5. Konecny G, Pauletti G, Pegram M, Untch M, Dandekar S, Aguilar Z, et al. Quantitative association between HER-2/neu and steroid hormone receptors in hormone receptor-positive primary breast cancer. J Natl Cancer Inst 2003;95:142-53.

6. Shin HY, Kim SH, Lee YJ, Kim DK. The effect of mechanical ventilation tidal volume during pneumoperitoneum on shoulder pain after a laparoscopic appendectomy. Surg Endosc 2010;24:2002-7.

7. Park S, Koo JS, Kim MS, Park HS, Lee JS, Lee JS, et al. Characteristics and outcomes according to molecular subtypes of breast cancer as classified by a panel of four biomarkers using immunohistochemistry. Breast 2012;21:50-7.

8. Eroles P, Bosch A, Perez-Fidalgo JA, Lluch A. Molecular biology in breast cancer: intrinsic subtypes and signaling pathways. Cancer Treat Rev 2012;38:698-707.

9. Prat A, Pineda E, Adamo B, Galvan P, Fernandez A, Gaba L, et al. Clinical implications of the intrinsic molecular subtypes of breast cancer. Breast 2015;24:S26-35.

10. Filipits M, Nielsen TO, Rudas M, Greil R, Stoger H, Jakesz R, et al. The PAM50 risk-of-recurrence score predicts risk for late distant recurrence after endocrine therapy in postmenopausal women with endocrine-responsive early breast cancer. Clin Cancer Res 2014;20: 1298-305.

11. Vaz-Luis I, Ottesen RA, Hughes ME, Marcom PK, Moy B, Rugo HS, et al. Impact of hormone receptor status on patterns of recurrence and clinical outcomes among patients with human epidermal growth factor-2-positive breast cancer in the national comprehensive cancer network: a prospective cohort study. Breast Cancer Res 2012;14:R129.

12. Perez EA, Romond EH, Suman VJ, Jeong JH, Davidson NE, Geyer $\mathrm{CE}$, Jr., et al. Four-year follow-up of trastuzumab plus adjuvant chemotherapy for operable human epidermal growth factor receptor 2-positive breast cancer: joint analysis of data from NCCTG N9831 and NSABP B-31. J Clin Oncol 2011;29:3366-73.

13. Houston SJ, Plunkett TA, Barnes DM, Smith P, Rubens RD, Miles DW. Overexpression of c-erbB2 is an independent marker of resistance to endocrine therapy in advanced breast cancer. $\mathrm{Br} \mathrm{J}$ Cancer 1999;79:1220-6

14. Andrulis IL, Bull SB, Blackstein ME, Sutherland D, Mak C, Sidlofsky $\mathrm{S}$, et al. neu/erbB-2 amplification identifies a poor-prognosis 
group of women with node-negative breast cancer. Toronto breast cancer study group. J Clin Oncol 1998;16:1340-9.

15. Kim HS, Park I, Cho HJ, Gwak G, Yang K, Bae BN, et al. Analysis of the potent prognostic factors in luminal-type breast cancer. J Breast Cancer 2012;15:401-6.

16. Park K, Han S, Kim JY, Kim HJ, Kwon JE, Gwak G. Silver-enhanced in situ hybridization as an alternative to fluorescence in situ hybridization for assaying HER2 amplification in clinical breast cancer. J Breast Cancer 2011;14:276-82.

17. Goldhirsch A, Ingle JN, Gelber RD, Coates AS, Thürlimann B, Senn HJ, et al. Thresholds for therapies: highlights of the St Gallen international expert consensus on the primary therapy of early breast cancer 2009. Ann Oncol 2009;20:1319-29.

18. Viani GA, Afonso SL, Stefano EJ, De Fendi LI, Soares FV. Adjuvant trastuzumab in the treatment of her-2-positive early breast cancer: a meta-analysis of published randomized trials. BMC Cancer 2007;7: 153.

19. Goss PE, Ingle JN, Pritchard KI, Robert NJ, Muss H, Gralow J, et al. Extending aromatase-inhibitor adjuvant therapy to 10 years. N Engl J Med 2016;375:209-19.

20. Onitilo AA, Engel JM, Greenlee RT, Mukesh BN. Breast cancer subtypes based on ER/PR and Her2 expression: comparison of clinicopathologic features and survival. Clin Med Res 2009;7:4-13.

21. Negi P, Kingsley PA, Jain K, Sachdeva J, Srivastava H, Marcus S, et al.
Survival of triple negative versus triple positive breast cancers: comparison and contrast. Asian Pac J Cancer Prev 2016;17:3911-6.

22. Kollias J, Elston CW, Ellis IO, Robertson JF, Blamey RW. Early-onset breast cancer-histopathological and prognostic considerations. Br J Cancer 1997;75:1318-23.

23. Jack RH, Davies EA, Renshaw C, Tutt A, Grocock MJ, Coupland $\mathrm{VH}$, et al. Differences in breast cancer hormone receptor status in ethnic groups: a London population. Eur J Cancer 2013;49:696702 .

24. Inwald EC, Klinkhammer-Schalke M, Hofstädter F, Zeman F, Koller M, Gerstenhauer M, et al. Ki-67 is a prognostic parameter in breast cancer patients: results of a large population-based cohort of a cancer registry. Breast Cancer Res Treat 2013;139:539-52.

25. Hashmi AA, Aijaz S, Khan SM, Mahboob R, Irfan M, Zafar NI, et al. Prognostic parameters of luminal A and luminal B intrinsic breast cancer subtypes of Pakistani patients. World J Surg Oncol 2018;16:1.

26. Bilalovic N, Vranic S, Hasanagic S, Basic H, Tatarevic A, Beslija S, et al. The Bcl-2 protein: a prognostic indicator strongly related to ER and PR in breast cancer. Bosn J Basic Med Sci 2004;4:5-12.

27. Thomadaki H, Talieri M, Scorilas A. Prognostic value of the apoptosis related genes BCL2 and BCL2L12 in breast cancer. Cancer Lett 2007;247:48-55 